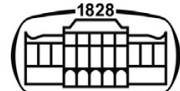

AKADÉMIAI KIADÓ

Acta Chromatographica

34 (2022) 1, 71-76

DOl:

$10.1556 / 1326.2020 .00882$

(C) 2020 The Author(s)

\section{ORIGINAL RESEARCH} PAPER

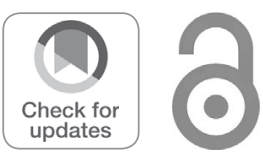

\title{
Identification and quantification of fatty acids in hunting web of adult Steatoda grossa (Theridiidae) female spiders
}

\author{
DŻASTIN ZIMNY ${ }^{1}$, MICHAŁ PATRZAŁEK ${ }^{2}$, \\ TERESA KOWALSKA ${ }^{1}$, MIECZYSŁAW SAJEWICZ ${ }^{1}$, \\ KINGA SURMIAK-STALMACH ${ }^{3}$ and GRAŻYNA WILCZEK ${ }^{3^{*}}$ ๑ \\ ${ }^{1}$ Institute of Chemistry, University of Silesia, Szkolna 9, Katowice 40-006, Poland \\ ${ }^{2}$ ICB Pharma, Stanislawa Lema 10, Jaworzno 43-603, Poland \\ ${ }^{3}$ Institute of Biology, Biotechnology and Environmental Protection, University of Silesia, Bankowa 9, \\ Katowice 40-007, Poland
}

Received: December 7, 2020 • Accepted: December 22, 2020

Published online: January 23, 2021

\begin{abstract}
This is the first study on composition of fatty acids in hunting web of Steatoda grossa (Theridiidae) spiders and one of only four similar studies ever made. Its main contribution is a discovery that fatty acids not only cover an outside of the web fibers, but they are even more abundantly represented in the fibers' inner structure. Although little attention has been so far attributed to the contents of fatty acids in spider silks, one has to remember that their biocompatibility combined with an extraordinary tensile strength make them a worth investigating template for material bioengineering studies.
\end{abstract}

\section{KEYWORDS}

spider silk, Steatoda grossa spiders, fatty acids composition, fatty acids distribution, material bioengineering template

\section{INTRODUCTION}

First suggestion on a possibility of having fatty acids included in spider silk was made in 1991 [1], although at that time no experimental evidence has yet been provided. Ten years later, Schulz produced the first evidence on the presence of lipids (i.e., the straight and branched alkanes, alkanols, alkanediols, fatty acids and glyceryl ethers) in spider silk of the Nephila clavipes spider [2]. The author had at his disposal considerable portions (up to $2 \mathrm{~g}$ per one batch) of spider silk collected from the female $N$. clavipes spiders specially bred for the purpose. First, that silk has been soaked for several hours in dichloromethane $\left(\mathrm{CH}_{2} \mathrm{Cl}_{2}\right)$ and then the extract underwent a rather complex derivatization procedure to ultimately result in the solution of fatty acid methyl esters in $n$-pentane. Finally, the analyses were performed by means of gas chromatography-mass spectrometry (GC-MS) and a statement was issued that fatty acids predominating in the investigated samples were palmitic acid (39\% of all acids), stearic acid (15\% of all acids) and the two double unsaturated C18 fatty acids, i.e., linoleic and linolelaidic acid ( $8 \%$ of all acids). Moreover, the presence of a vast selection of other saturated and unsaturated fatty acids with an even and odd number of carbon atoms from C12 to C24 was also confirmed, which contributed from one to several percent each to an overall fatty acids pool. A remark was added that the contents of fatty acids were the rough estimates only, due to perceptible differences in composition from one spider silk batch to another. Certain drawback of this otherwise extensive and informative report was that identification of individual fatty acids was not direct (i.e., not based on a comparison of their retention times 
and mass spectra with those of respective standard compounds). Instead, the author largely based an identification approach on gas chromatographic retention indices.

In 2008, a paper was published on specificity of spider silk composition depending on life cycles of female Coelotes terrestris spiders and more specifically, on their social (i.e., parental) and solitary (i.e., the postdispersal young to the adult stage) life periods [3]. The silk samples were soaked for $15 \mathrm{~min}$ in $n$-pentane and the analysis of the resulting extracts upon derivatization of fatty acids to their methyl esters was performed by means of gas chromatography-mass spectrometry (GC-MS). Identification of individual chemical species was carried out with use of fatty acid standards. Depending on different life periods, different proportions of four predominant fatty acids in the spider silk of the $C$. terrestris spiders were documented, which were palmitic, stearic, linoleic, and oleic acid.

In 2008, a review paper was published on an elaborate structure of spider silk [4], which despite of a promising title and rather abundant information regarding amino acids and peptides contained in spider silk, offered but a laconic mention that fatty acids also contribute to spider silk, supported by just one outdated reference [1].

In 2019, one more paper was published by the Pakistani authors on the analysis of fatty acids in spider silk from an undefined spider species, collected from a natural roadside habitat in the Pakistani Lahore district [5]. Relatively high aliquots of spider silk ( $2 \mathrm{~g}$ per one batch) were then manually cleaned and extracted for 3 hours with $n$-hexane in the Soxhlet apparatus. After that time, all organic matter isolated in the extraction process was esterified and the obtained esters were separated and identified by means of gas chromatography with the flame ionization detection (GC-FID). Identification of individual fatty acids was carried out with support of the standards and similar to the results provided in paper [2], presence of 14 saturated and unsaturated fatty acids with even and odd carbon atom numbers from C8 to C24 was confirmed, with individual compounds being quantified.

Extraction of spider silk and further processing of the extract to obtain fatty acid methyl esters apparently is a fairly sensitive issue. The main reason is that one can never be sure if the detected compounds are a genuine spider product meant to coat the silk fibers, in despite of the fact that the silk samples collected for the analysis need to be very carefully cleaned from the animals' diet and defecation leftovers, prior to the analysis proper. Maybe this is one reason why the available literature on fatty acids present in spider silk is very scant, as confirmed by just three experimental studies $[2,3,5]$ reported in the course of the pasts three decades. Another comment has to be added that the investigations carried out so far have focused exclusively on these fatty acids that coat the web silk from an outside only and could easily be flushed with non-polar solvents (like, e.g., $n$-pentane, $n$-hexane, or dichloromethane). These observations instigated our interest in composition of fatty acids not only externally coating spider silk of the Steatoda grossa spider, but also of those imbedded inside the silk fibers which cannot simply be flushed. In that way, we extended our interest in chemical composition of the S. grossa web silk, initiated several years ago with the thin-layer chromatographic examination of the amino acid profile of the silk originating from the same animal species [6,7]. The results of our current study carried out with use of gas chromatography with two types of detection systems (FID: flame ionization detection, and MS: mass spectrometric detection) on composition of fatty acids contained outside and inside the spider silk fibers are going to be presented in the forthcoming sections.

\section{MATERIAL AND METHODS}

\section{Spider silk samples and their collection and preparation}

The study was carried out on tangled, three-dimensional "cobweb" snare (Fig. 1A) produced by adult females of the $S$. grossa (Theridiidae) spiders originating from a multi-generation experimental laboratory colony. Hunting webs woven by adult specimens comprise mainly dry supporting threads (Fig. 1B), only fragmentarily covered by the glue regions (Fig. 1C) and sticky gumfooted threads, including sticky silk, which adheres the supporting threads to the surfaces $[8,9]$.

Spiders were kept separately in plastic boxes $(9.5 \times 7.5$ $\times 5.5 \mathrm{~cm}$ ) with wooden frames, supporting spinning of hunting webs. The boxes with spiders were placed in breeding chambers kept in standard conditions of light/dark cycle $(12 \mathrm{~L}: 12 \mathrm{D})$, temperature $\left(\mathrm{L}: 27^{\circ} \mathrm{C}, \quad \mathrm{D}: 18^{\circ} \mathrm{C}\right)$ and humidity $(70 \pm 10 \%)$. The spiders every 2 days were fed 2-3 Drosophila hydei flies, which grown on standard medium
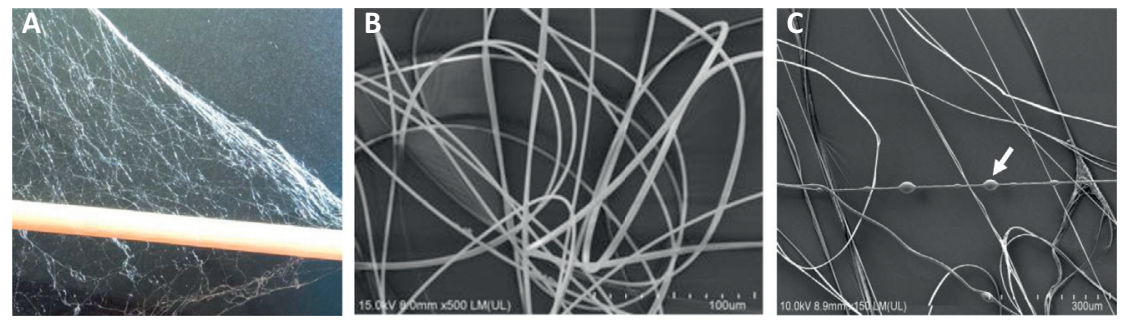

Fig. 1 Hunting web of S. grossa females. A. Image of webs woven on wooden frame. B. SEM images of dry web threads C. SEM images of web threads with glue regions 
[10]. Analysis involved the dry regions of both supporting threads and sticky gumfooted threads (Fig. 1). To measure the analyzed parameters, a total of 200 hunting webs were collected.

\section{Esterification of fatty acids contained in spider silk samples}

In this study, four different types of the spider silk samples were used: (i) not cleaned, (ii) manually cleaned under a binocular microscope from food leftovers and excrements, (iii) not cleaned and hydrolyzed with $\mathrm{HCl}$, and (iv) manually cleaned under a binocular microscope and hydrolyzed with $\mathrm{HCl}$. Prior to esterification, the 4-mg silk samples (i) and (ii) were washed with $2.0 \mathrm{~mL} n$-hexane by an energetic shaking to dissolve fatty acids present on the silk surface. The obtained $n$-hexane solutions underwent esterification. Samples (iii) and (iv) (the 4-mg aliquot each) were first hydrolyzed with $8 \mathrm{~mL} 6 \mathrm{M}$ hydrochloric acid (PPH POCh, Gliwice, Poland) [6] and to the obtained hydrolyzates, $4 \mathrm{~mL}$ $n$-hexane was added and the entity was energetically shaken to transfer fatty acids from the aqueous to organic phase. After phase separation, the $n$-hexane layers underwent esterification.

Esterification was carried out in the round-bottomed flask, in which $2.00 \mathrm{~mL} n$-hexane solution of fatty acids, 3.75 $\mathrm{ml}$ methanol, and $0.75 \mathrm{~mL} 8 \% \mathrm{HCl}$ was placed, and the entity was kept for $45 \mathrm{~min}$ at the boiling point under the reflux. After that time, the contents of the flask was cooled and transferred to the separation funnel, and the flask was rinsed with $1 \mathrm{~mL} n$-hexane and $1 \mathrm{~mL}$ water (which were eventually pooled with the post-esterification product). The contents of the funnel was energetically shaken for $10 \mathrm{~min}$ and then left for phase separation. The separated organic layer was placed in a vial and the solvent was evaporated in a delicate stream of air. At the end, $10 \mu \mathrm{L} n$-hexane was added to the vial, to obtain solution of the esterified fatty acids mixture for the gas chromatographic analyses and the $2-\mu \mathrm{L}$ aliquots of this solution were used for the analyses.

\section{Gas chromatographic working conditions}

Gas chromatographic analyses were carried out with use of the two gas chromatographic systems (A) and (B), and the respective working conditions are given below:

System (A): For the qualitative analysis, the ThermoQuest CE Instruments Trace GC 2000 series capillary gas chromatograph with the Finnigan Trace MS detector (Thermo Electron Corporation, Austin, TX, USA) and the on-column injection was used and the analyses were performed on the fused silica Quadrex capillary column 5MS (Quadrex Corporation, Woodbridge, CT, USA). Column length $(l)=30 \mathrm{~m}$, inner diameter (i.d.) $=0.32 \mathrm{~mm}$, stationary phase film thickness $\left(d_{f}\right)=0.5 \mu \mathrm{m}$. Helium $(\mathrm{He})$ as carrier gas was used at the flow rate of $5 \mathrm{~mL} \mathrm{~min}{ }^{-1}$. The temperature gradient mode: $2 \mathrm{~min}$ at $100^{\circ} \mathrm{C}$; temperature rise to $150^{\circ} \mathrm{C}$ at the rate $10^{\circ} \mathrm{C} \mathrm{min}^{-1} ; 1 \mathrm{~min}$ at $150^{\circ} \mathrm{C}$; temperature rise to $280^{\circ} \mathrm{C}$ at the rate $15^{\circ} \mathrm{C} \mathrm{min}{ }^{-1} ; 20 \mathrm{~min}$ at $280^{\circ} \mathrm{C}$; temperature of the injector at splitless mode, $280^{\circ} \mathrm{C}$.
Moreover, the discussed chromatographic system is equipped with the NIST mass spectral library (National Institute of Standards and Technology, Gaithersburg, MD, USA), to facilitate identification of separated analytes based on the mass spectra. The retention time $\left(t_{\mathrm{R}}\right)$ values for methyl esters of the following fatty acids were: capric acid, $7.10 \mathrm{~min}$; lauric acid, $10.05 \mathrm{~min}$; myristic acid, $12.08 \mathrm{~min}$; palmitic acid, $13.62 \mathrm{~min}$; stearic acid, $15.00 \mathrm{~min}$.

System (B): For quantitative analysis, the Fisons Instruments GC 8000 Series capillary gas chromatograph with the on-column injection and flame ionization detection (FID) (Fisons Instruments, Milan, Italy) was used, and the analyses were carried out on the Chromapack CP-Sil 8CB capillary column (Agilent, Santa Clara, CA, USA). Column length $(l)=50 \mathrm{~m}$, inner diameter (i.d.) $=0.53 \mathrm{~mm}$, stationary phase film thickness $\left(d_{f}\right)=1 \mu \mathrm{m}$. Helium $(\mathrm{He})$ as carrier gas was used at the flow rate of $4 \mathrm{~mL} \mathrm{~min}^{-1}$. The temperature gradient mode: $3 \mathrm{~min}$ at $100^{\circ} \mathrm{C}$; temperature rise to $170^{\circ} \mathrm{C}$ at the rate $10^{\circ} \mathrm{C} \mathrm{min}^{-1}$; temperature rise to $200^{\circ} \mathrm{C}$ at the rate $20^{\circ} \mathrm{C} \mathrm{min}^{-1} ; 5 \mathrm{~min}$ at $200^{\circ} \mathrm{C}$; temperature

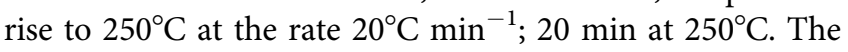
injector temperature was set at $300^{\circ} \mathrm{C}$. The retention time $\left(t_{\mathrm{R}}\right)$ values for methyl esters of the following fatty acids were: capric acid, $9.50 \mathrm{~min}$; lauric acid, $12.00 \mathrm{~min}$; myristic acid, $15.60 \mathrm{~min}$; palmitic acid, $18.21 \mathrm{~min}$; stearic acid, $21.00 \mathrm{~min}$.

\section{Qualitative and quantitative analysis of fatty acids contained in spider silk samples}

The GC-MS analytical system (A) was used to confirm an identity of the five targeted fatty acids contained in the four spider silk samples by (i) a comparison of their respective retention time $\left(t_{\mathrm{R}}\right)$ values with those of the ME10-KT kit of the fatty acid methyl ester (FAME) standards (solutions in $n$-hexane at the concentration of $1,000 \mu \mathrm{g} \mathrm{mL} \mathrm{m}^{-1}$ each; Merck, Darmstadt, Germany) and (ii) a comparison of their mass spectra with those contained in the virtual NIST mass spectral library of the GC-MS instrument.

The GC-FID analytical system (B) was also used to confirm an identity of the five targeted fatty acids contained in the four spider silk samples by a comparison of their respective retention time $\left(t_{\mathrm{R}}\right)$ values with those of the ME10-KT kit of the fatty acid methyl ester (FAME) standards. Moreover, system (B) was used for quantitative analysis of the five targeted fatty acids. Because of low amounts of the spider silk samples (4 mg per one sample type) in our disposal, only these fatty acids were quantified, which appeared on the chromatograms above their respective quantification levels. Calibration curves for the selected fatty acids were performed with aid of the FAME standards by their injection to the GC-FID system in the 1-, 2-, 3-, 4-, and $5-\mu \mathrm{L}$ aliquots in triplicate $(n=3)$. Respective levels of detection (LOD) and levels of quantification (LOQ) were calculated from the following relationships: LOD $=3.3 \times$ $\mathrm{SD} / a$ and $\mathrm{LOQ}=10 \times \mathrm{SD} / a$, where $\mathrm{SD}$ is the standard deviation of the peak height taken as the noise measure, and $a$ is the slope of the corresponding calibration curve ( $\mathrm{y}=a \mathrm{x}$ $+b)$. The obtained results are given in Table 1 . 
Table 1. Retention time $\left(t_{\mathrm{R}}\right)$ values, calibration curves, and the LOD and LOQ values obtained for standard samples of the investigated fatty acid methyl esters in the employed GC-FID system $(n=3)$

\begin{tabular}{lccccc}
\hline Fatty acid & $\begin{array}{c}\text { Retention time, } \\
t_{\mathrm{R}}[\mathrm{min}]\end{array}$ & Calibration curve & $\begin{array}{c}\text { Correlation } \mathrm{c} \\
\text { oefficient, } r\end{array}$ & $\begin{array}{c}\text { LOD }\left[\mu \mathrm{g} \mu \mathrm{L}^{-1}\right] \\
(\mathrm{RSD}[\%])\end{array}$ & $\begin{array}{c}\mathrm{LOQ}\left[\mu \mathrm{g} \mu \mathrm{L}^{-1} \text { ] }\right. \\
(\mathrm{RSD}[\%])\end{array}$ \\
\hline Capric acid, C10:0 & 9.50 & $\mathrm{y}=126.92 \mathrm{x}-19.62$ & 0.992 & $0.007(4.95)$ & $0.022(4.95)$ \\
Lauric acid, C12:0 & 12.00 & $\mathrm{y}=147.98 \mathrm{x}-34.84$ & 0.990 & $0.006(3.75)$ & $0.019(3.75)$ \\
Myristic acid, C14:0 & 15.60 & $\mathrm{y}=142.88 \mathrm{x}-4.48$ & 0.999 & $0.006(3.92)$ & $0.019(3.92)$ \\
Palmitic acid, C16:0 & 18.21 & $\mathrm{y}=65.3 \mathrm{x}-10.01$ & 0.999 & $0.014(2.98)$ & $0.043(2.98)$ \\
Stearic acid, C18:0 & 21.00 & $\mathrm{y}=36.6 \mathrm{x}-4.12$ & 0.997 & $0.021(4.25)$ & $0.064(4.25)$ \\
\hline
\end{tabular}

Table 2. Contents of capric acid, lauric acid, myristic acid, palmitic acid, and stearic acid in spider silk of the S. grossa spider prepared for the analysis in four different ways: (i) sample not cleaned, washed with $n$-hexane; (ii) sample mechanically cleaned, washed with $\mathrm{n}$-hexane;

(iii) sample not cleaned, hydrolyzed with $\mathrm{HCl}$; and (iV) sample mechanically cleaned, hydrolyzed with $\mathrm{HCl}(n=3)$

\begin{tabular}{lccccrr}
\hline & & \multicolumn{5}{c}{ Content of fatty acid $[\mu$ g fatty acid per $1 \mathrm{mg}$ spider silk] (RSD [\%]) } \\
\cline { 3 - 7 } No. & Silk sample & Capric acid, C10:0 & Lauric acid, C12:0 & Myristic acid, C14:0 & Palmitic acid, C16:0 & Stearic acid, C18:0 \\
\hline 1 & (i) & n.d. & n.d. & $0.36(5.24)$ & $4.81(4.74)$ & $2.59(6.88)$ \\
2 & (ii) & $0.04(5.64)$ & $0.06(7.12)$ & $0.30(4.14)$ & $3.05(2.87)$ & $0.73(5.39)$ \\
3 & (iii) & $0.46(3.98)$ & $0.19(5.98)$ & $0.55(3.99)$ & $10.62(6.69)$ & $14.41(2.77)$ \\
4 & (iv) & $0.67(6.14)$ & $0.07(4.87)$ & $0.41(6.38)$ & $5.67(4.98)$ & $8.46(4.13)$ \\
\hline
\end{tabular}

n.d.: not detected.

Quantification was performed for the 2- $\mu \mathrm{L}$ aliquots of the esterified spider silk samples analyzed in triplicate $(n=3)$ and the obtained results are given in Table 2.

\section{RESULTS AND DISCUSSION}

An inspiration for this research was our awareness of remarkable properties of spider silk, and in the first instance, of its outstanding tensile strength (higher than that of steel and Kevlon) and its potential biocompatibility, thus making it an interesting template for material bioengineering studies. However, there is careful analysis of chemical composition and structure of spider silk needed as an inevitable precondition to help us fully understand its amazing physical properties and at a subsequent step, to develop synthetic materials imitating this natural one. So far, certain attention has been paid to amino acids, peptides and proteins included in the spider web structure $[6,7,11-16]$, but the entity of structural components (including fatty acids) still remains quite neglected.

The main novelty in our approach to identification and quantification of fatty acids contained in spider silk is that we attempted to answer a very serious analytical question on how these compounds are distributed across the spider silk fibers. An idea standing behind the experiments reported so far $[2,3,5]$ was that fatty acids externally coat these fibers and hence, that they can easily be washed from the web by soaking it in the low-polar organic solvents (like $n$-pentane, or $n$-hexane). We extended the search for fatty acids to the deeper silk fiber layers and to this effect we had our samples divided into four categories. Samples from the first two categories ((i) native silk and (ii) native silk manually cleaned from contamination with food leftovers and excrements) were washed with $n$-hexane, to retrieve fatty acids externally coating the fibers. Samples from categories (iii) (native silk digested in $\mathrm{HCl}$ ) and (iv) (native silk manually cleaned and then digested in $\mathrm{HCl}$ ) contained both, fatty acids from the surface coating and also those which are deeper imbedded in the web structure. With help of the employed fatty acid methyl ester (FAME) standards, the developed calibration curves, and an additional confirmation of identity with aid of the NIST mass spectral library, we managed to identify and quantify each fatty acid in the investigated samples.

Five fatty acids detected and quantified in this study (i.e., capric (C10:0), lauric (C12:0), myristic (C14:0), palmitic (C16:0) and stearic (C18:0) acid) have also been detected and quantified in a surface coating of the $N$. clavipes spider fibers [2] and in a surface coating of an unnamed spider genus from Pakistan [5]. Absolute predominance of palmitic and stearic acid in an external coating of the C. terrestris spider web has been reported in paper [3], and this regularity holds for the other investigated spider species as well $[2,5]$.

The results presented in Table 2 and valid for the silk of the $S$. grossa spider also show distinct predominance of palmitic and stearic acid among the five fatty acids discussed in this study. In general, the contents of capric, lauric, and myristic acid in all samples are by one or two magnitude orders lower than those of palmitic and stearic acid. What is even more interesting, the contents of all fatty acids in the hydrolyzed samples (both not cleaned and cleaned) are in most cases significantly higher than in those washed with $n$-hexane only. Among the most spectacular examples of such disproportion, one can point out to differences between the contents of capric acid in samples (i) and (iii) (not 
detected in (i) vs. $0.46 \mu \mathrm{g} \mathrm{mg}^{-1}$ in (iii)), or between the contents of stearic acid in samples (i) and (iii) $\left(2.59 \mu \mathrm{g} \mathrm{mg}^{-1}\right.$ in (i) vs. $14.41 \mu \mathrm{g} \mathrm{mg}^{-1}$ in (iii)). Thus a justified conclusion can be drawn that significant amounts of fatty acids cannot be just washed out from an outer surface of spider silk because they are imbedded in its inner structures which is not penetrated by the solvent and they can only be retrieved through hydrolytic destruction of the fibers.

Manual pre-treatment (i.e. cleaning) of spider silk prior to further analytical steps contains a considerable component of randomness and hence, it is hardly possible to conclude as to its influence on the ultimate analytical result. However, in the seven out of ten cases the contents of respective fatty acids in the manually cleaned spider silk samples were lower than in the not cleaned ones (see Table 2). Thus it can only be speculated that from the food leftovers and excrements contaminating the not cleaned web additional amounts of fatty acids might have been extracted. An alternative and probably less convincing explanation might also be that in the process of manual cleaning certain amounts of fatty acids were involuntarily removed as well.

Finally, one has to emphasize that within the framework of the method developed in this study, we can specify the amounts of each individual fatty acid per $1 \mathrm{~g}$ of the silk web (see Table 2), which has not been presented in papers $[2,3,5]$. With palmitic and stearic acid, each of these two compounds contributes from slightly below to slightly above $1 \%$ of the overall spider silk weight, depending on the analyzed sample. In the case of capric, lauric, and myristic acid, their contributions to an overall spider silk weight always are below $1 \mu \mathrm{g}$ fatty acid per $1 \mathrm{mg}$ spider silk, i.e., all of them remain below the per mille level.

\section{CONCLUSIONS}

Within the framework of this study, identification and quantification of five predominant fatty acids (i.e., capric, lauric, myristic, palmitic, and stearic acid) in spider silk of the adult S. grossa (Theridiidae) female spiders was performed. Basic achievements of this study can be summarized, as follows:

- This is first report on composition of fatty acids in the $S$. grossa spider silk;

- This is also first report on composition of fatty acids in spider silk originating from a clearly defined web type (i.e., the hunting web), while in all previous papers on a similar subject matter, the web types have never been identified and it could easily be assumed that those samples represented mixtures of different web types;

- For the first time, an assumption was made that fatty acids not only externally coat the spider silk fibers, but they are also imbedded in their inner structure and can be retrieved from an inside through hydrolysis alone;

- The quantity of fatty acids contained in spider silk was for the first time examined and given in micrograms per $1 \mathrm{mg}$ spider web;
- It was established that the sum of five predominant fatty acids from the outer and inner parts of hunting web of the adult $S$. grossa (Theridiidae) female spiders was below $30 \mu \mathrm{g}$ per $1 \mathrm{mg}$ spider silk, independent of the sample type considered ((a) $\div(\mathrm{d}))$. Thus a justified assumption could be drawn that the totality of fatty acids (including those appearing in trace amounts and not quantified in this study) cannot surpass $3 \%$ of the total spider silk weight.

\section{ACKNOWLEDGMENTS}

We thank Dr Jagna Karcz for technical help in SEM analyzes and images preparation. This work was financially supported by the Ministry of Science and Higher Education: Statutory activity (subsidies for 2020).

\section{REFERENCES}

1. Vollrath, F., Tillinghast E. K. Glycoprotein glue beneath a spider's web. Naturwissenschaften 1991, 78, 557-9.

2. Schulz, S. Composition of the silk lipids of the spider Nephila clavipes. Lipids 2001, 36, 637-47.

3. Trabalon, M., Assi-Bessekon, D. Effects of web chemical signatures on intraspecific recognition in a subsocial spider, Coelotes terrestris (Araneae). Anim. Behav. 2008, 76, 1571-8.

4. Römer, L., Scheibel, T. The elaborate structure of spider silk. Prion 2008, 2, 154-61.

5. Iqbal, Z., Liaqat, M., Shoukat, A., Usmam Sabri, M., Akhtar, M. Fatty acid profile of web silk from Pakistan. World J. Pharm. Res. 2019, 8, 110-8.

6. Michalik, M., Surmacka, M., Stalmach, M., Wilczek, G., Kowalska, T., Sajewicz, M. Application of TLC to ecotoxicological study with the Steatoda grossa spider web. J. Planar Chromatogr. - Mod. TLC 2018, 31, 7-12.

7. Wilczek, G., Surmiak, K., Wawszczak, B., Sajewicz, M., Kowalska, T., Sindera, P., Wiśniewska, K., Szulińska, E. Effect of long-term cadmium and copper intoxication of on the efficiency of ampullate silk glands in false black widow Steatoda grossa (Theridiidae) spiders. Comp. Biochem. Physiol. Part C: Toxicol. Pharmacol. 2019, 224, 108564.

8. Benjamin, S. P., Zschokke, S. Webs of theridiid spiders: construction, structure and evolution. Biol. J. Linn. Soc. 2003, 78(3), 293-305.

9. Blackledge, T. A., Zevenbergen, J. N. Condition dependent spider web architecture in the western black widow, Latrodectus hesperus. Anim. Behav. 2007, 73, 855-64.

10. Shukla, A. K., Pragya, P., Kar Chowdhuri, D. A modified alkaline Comet assay for in vivo detection of oxidative DNA damage in Drosophila melanogaster. Mutat. Res. 2011, 726, 222-6.

11. Lombardi, S. J., Kaplan, D. L. The amino acid composition of major ampullate gland silk (dragline) of Nephila clavipes (Araneae, Tetragnathidae). J. Arachnol 1990, 18, 297-306. 
12. Römer, L., Scheibel, T. The elaborate structure of spider silk. Prion 2008, 2, 154-61.

13. Zhang, Y., Dai, L., Ou-Yang, Z.-C. Amino-acid-dependent elasticity of spider silk. Int. J. Mod. Phys. B 2004, 18, 2516-22.

14. Savage, K. N., Gosline, J. M. The effect of proline on the network structure of major ampullate silks as inferred from their mechanical and optical properties. J. Exp. Biol. 2008, 211, 1937-47.
15. Savage, K. N., Gosline, J. M. The role of proline in the elastic mechanism of hydrated spider silks. J. Exp. Biol. 2008, 211, 1948-57.

16. Garb, J. E., Haney, R. A., Schwager, E. E., Gregorič, M., Kuntner, M., Agnarsson, I., Blackledge, T. A. The transcriptome of Darwin's bark spider silk glands predicts proteins contributing to dragline silk toughness. Commun. Biol. 2019, 2, 275. 